\title{
Amino Acid Contents and Glutamate Decarboxylase Activity in Brain of Methylazoxymethanol-induced Microencephalic Rats*
}

\author{
Yukio NaKamura, Yukio Nagata, Masahiko Kato, \\ Atsuko OKUMURA and Fumiaki SuzUKI \\ Department of Agricultural Chemistry, Faculty of Agriculture, Gifu University, \\ Naka, Kagamigahara 504, Japan \\ Received August 27, 1979
}

\begin{abstract}
The amounts of amino-acid neurotransmitters and GAD activity were determined in different brain regions of microencephalic rats that were offspring of mother rats injected with methylazoxymethanol (MAM)-acetate on the 15 th day of pregnancy.

The contents of GABA, taurine, aspartic acid, glutamic acid, and glycine in the cerebrum, the target organ of MAM, of 90 days old MAM-induced microencephalic rats were higher than those of control animals. No difference was noted in the contents of these neurotransmitters on postpartum day 21 except for aspartic acid. The postnatal contents of these amino-acid neurotransmitters in the cerebellum, which is not a target organ of MAM, were not affected by prenatal MAM-treatment, except that GABA in 21 days old MAM-induced microencephalic rats was lower than in control rats.

In microencephalic rats, the cerebral cortex, nucleus caudate-putamen, hippocampus, and thalamus-hypothalamus weighed $50 \%$ less than in control rats, but the colliculus-midbrain, and cerebellum had similar weights to control rats.

GAD activity per gram wet weight of cerebrum increased rapidly for 21 days after birth, and then increased gradually. No difference was found in GAD activity in MAM-induced microencephalic and control rats. The results are discussed in connection with the biochemical adaptability of neurons.
\end{abstract}

MAM is an aglycone of cycasin that was isolated from cycard plants, ${ }^{2)}$ and is hepatotoxic, ${ }^{3)}$ carcinogenic, ${ }^{4)}$ and neurotoxic. ${ }^{5)}$ Weight of cerebral hemisphere of offspring of the pregnant rats which were injected with MAM in the beginning of the third trimester of pregnancy was significantly lower than that of normal animals. Learning ability of MAMinduced microencephalic rats was inferior to that of control animals. The extent of inferiority, however, was not so large as the degree of reduction in telencephalic mass, and their behaviour seemed normal. ${ }^{6)}$ The result suggests that some mechanism compensates the decrease in the number of neurons in the

* Biochemical Studies on the Brain of a Methylazoxymethanol-induced Microencephalic Rat. For Part II, see ref. 1 .

Abbreviations: MAM, methylazoxymethanol; GAD, glutamate decarboxylase (EC 4.1.1.15), GABA, $\gamma$-aminobutyric acid. central nervous system of the microencephalic rat.

Excitation conduced in a neuron must be transmitted to other neuron. For the purpose of transmission is a synapse formed in which an ending of an axon connects to the next neuron. Transmission of nervous information is mediated in the region by some chemical transmitters, which have been proved or estimated to be cholines, amines and amino acids. ${ }^{7)}$

In the previous paper, ${ }^{1)}$ acetylcholinesterase activity per $g$ wet weight of the reduced cerebrum of a MAM-induced microencephalic rat was demonstrated to increase in proportion to the decrease in cerebral weight. As acetylcholinesterase activity in the brain is nearly parallel with acetylcholine concentration, we thought that the level of a cholinergic neurotransmitter increased in the reduced cerebrum. Contents of protein and 
DNA, which do not directly participate in nervous transmission, were not affected ${ }^{8,9}$; therefore only the substances participating in synaptic transmission were presumed to increase in compensation for reduction in cerebral mass.

In order to confirm this presumption, contents of other neurotransmitters (GABA, and other possible amino-acid neurotransmitters) and GABA-forming enzyme, GAD, were determined in the present study. Data about amine neurotrasmitters will be reported elsewhere.

\section{MATERIALS AND METHODS}

Animals. Rats of a Wistar strain used in this study were housed as reported previously. ${ }^{1)}$ Female rats being 15 days gone in pregnancy were injected intraperitoneally with $24 \mathrm{mg}$ of MAM-acetate in saline per $\mathrm{kg}$ of body weight. The offspring from the mothers were called MAMinduced microencephalic rats or simply microencephalic rats in this paper. The male microencephalic rats were used for determination of GAD activity and amino-acid neurotransmitters on day 21 and 90 after birth, whereas both males and females were used on day 0 and 10 . Experimental animals were weighed and decapitated at 10 a.m. The brain was rapidly removed and divided into 6 parts, (a) cerebral cortex, (b) nucleus caudatus and putamen, (c) hippocampus, (d) thalamus and hypothalamus, (e) colliculus and midbrain, and (f) cerebellum.

Assay of GAD activity. Each part of the brain was weighed immediately after removal of blood with filter paper, and was homogenized at $0^{\circ} \mathrm{C}$ for $5 \mathrm{~min}$ at 5 strokes in 9 volumes of $0.15 \mathrm{M}$ potassium chloride containing $5 \mathrm{mM}$ ethylendiaminetetraacetic acid with a Potter-Elvehjem homogenizer with a teflon pestle. The reaction mixture for determination of GAD activity contained $25 \mathrm{~mm}$ sodium glutamate, $0.5 \mathrm{~mm}$ pyridoxal phosphate, $0.1 \mathrm{M}$ phosphate buffer ( $\mathrm{pH} 6.4$ ), and $0.2 \mathrm{ml}$ of the homogenate in $1.0 \mathrm{ml}$ of total volume. After $5 \mathrm{~min}$ preincubation, the reaction was started by addition of the homogenate, carried out at $37^{\circ} \mathrm{C}$ for $30 \mathrm{~min}$, and terminated by addition of $0.25 \mathrm{ml}$ of $25 \%$ trichloroacetic acid. GABA formed by the enzymatic reaction was determined by the fluorescent spectrophotometric method. ${ }^{10)}$ One unit of GAD was defined as the amount of the enzyme which could form one $\mu \mathrm{mol}$ of GABA in 30 min under the experimental conditions.

Determination of amino-acid neurotransmitters. Each part of the brain was rapidly frozen in $75 \%$ ethanol which was previously cooled at $-60^{\circ} \mathrm{C}$ with dry ice-ethanol. The frozen tissue was weighed, and homogenized with $15 \mathrm{ml}$ of $75 \%$ ethanol per $g$ tissue. The homogenate was centrifuged at $10,000 \times g$ for $30 \mathrm{~min}$ at $-40^{\circ} \mathrm{C}$. The precipitate was suspended into $7.5 \mathrm{ml}$ of $75 \%$ ethanol per $g$ of the tissue, and centrifuged again. The supernatant was combined with the 1st supernatant, evaporated to dryness, and dissolved into $5 \mathrm{ml}$ of the citrate buffer ( $\mathrm{pH} 2.2)$. Being washed with 5 volumes of chloroform, the aqueous layer was used as the sample for determination of amino-acid neurotransmitters. They were determined with a JEOL Amino Acid Analyzer (JLC-6AH).

\section{RESULTS}

\section{Changes in the contents of possible amino-acid} neurotransmitters in the brain

Contents of GABA, taurine, aspartic acid, glutamic acid and glycine in the cerebrum of 21 and 90 days old rats are shown in Table I. A 90 days old MAM-induced microencephalic rat showed higher contents of all amino-acid

Table 1. Contents of Possible Amino-acid Neurotransmitters in the Cerebrum

\begin{tabular}{lcccc}
\hline Days after birth & & \multicolumn{2}{c}{90} \\
\hline & & \multicolumn{2}{c}{ Contents $(\mu \mathrm{mol} / \mathrm{g}$ wet weight $)$} \\
\cline { 2 - 5 } Amino acids & Control & Microencephalic & Control & Microencephalic \\
\hline GABA & $1.51 \pm 0.01$ & $1.61 \pm 0.12$ & $1.65 \pm 0.08$ & $2.36 \pm 0.13^{* * *}$ \\
Taurine & $9.59 \pm 0.35$ & $8.29 \pm 0.66$ & $3.88 \pm 0.24$ & $5.57 \pm 0.47^{* *}$ \\
Aspartic acid & $2.91 \pm 0.07$ & $2.47 \pm 0.09^{* *}$ & $1.82 \pm 0.06$ & $2.49 \pm 0.26^{*}$ \\
Glutamic acid & $9.48 \pm 0.15$ & $8.61 \pm 0.72$ & $8.23 \pm 0.16$ & $10.88 \pm 0.93^{* *}$ \\
Glycine & $0.47 \pm 0.03$ & $0.48 \pm 0.05$ & $0.56 \pm 0.01$ & $0.79 \pm 0.15^{*}$ \\
\hline
\end{tabular}

Each value represents the mean $\pm S D$ of 3 determinations. One litter ( $4 \sim 6$ rats) was used for one determination. Asterisks denote significant difference between control and microencephalic values: * significant at $P<0.05 ; *$ significant at $P<0.01 ; * * *$ significant at $P<0.001$. 
Table 11. Changes in GAD Activity in the Cerebrum during Development

\begin{tabular}{|c|c|c|c|c|}
\hline \multirow{3}{*}{$\begin{array}{c}\text { Days } \\
\text { after birth }\end{array}$} & \multicolumn{4}{|c|}{ GAD activity } \\
\hline & \multicolumn{2}{|c|}{ Units/g wet weight } & \multicolumn{2}{|c|}{ Units/cerebrum } \\
\hline & Control & Microencephalic & Control & Microencephalic \\
\hline 0 & $1.22 \pm 0.87(8)$ & $1.43 \pm 1.26(11)$ & $0.13 \pm 0.10(8)$ & $0.09 \pm 0.08(11)$ \\
\hline 10 & $9.62 \pm 4.19(7)$ & $8.62 \pm 2.23(6)$ & $6.55 \pm 2.85(7)$ & $2.43 \pm 0.63(6)^{* * *}$ \\
\hline 21 & $19.61 \pm 1.32(7)$ & $18.40 \pm 2.34(7)$ & $17.88 \pm 1.20(7)$ & $6.51 \pm 0.83(7)^{* * *}$ \\
\hline 90 & $27.06 \pm 2.80(8)$ & $26.00 \pm 1.54(7)$ & $28.38 \pm 2.94(8)$ & $12.24 \pm 0.73(7)^{* * *}$ \\
\hline
\end{tabular}

Each value represents the mean $\pm \mathrm{SD}$. The numbers of determinations are shown in parentheses. Asterisks denote significant difference between control and microencephalic values at $P<0.001$.

Table III. The Weight and GaD Activity in the Various Regions of the Brain of 90 Days OLd Rats

\begin{tabular}{|c|c|c|c|c|c|}
\hline \multirow{2}{*}{ Regions } & \multicolumn{2}{|c|}{ Wet weight $(\mathrm{g})$} & \multicolumn{2}{|c|}{ GAD activity (units/g wet weight) } & \multirow{2}{*}{$\begin{array}{c}\text { Total } \\
\text { GAD } \\
(\%)\end{array}$} \\
\hline & Control & Microencephalic & Control & Microencephalic & \\
\hline \multicolumn{6}{|l|}{ Cerebrum } \\
\hline Cerebral cortex & $0.689 \pm 0.065$ & $0.334 \pm 0.053^{* * *}$ & $18.8 \pm 1.6$ & $20.0 \pm 2.4$ & 51 \\
\hline $\begin{array}{l}\text { Nuc. caudate/ } \\
\text { Putamen }\end{array}$ & $0.139 \pm 0.016$ & $0.085 \pm 0.020^{*}$ & $23.0 \pm 3.2$ & $21.1 \pm 3.2$ & 56 \\
\hline Hippocampus & $0.149 \pm 0.018$ & $0.108 \pm 0.002^{* *}$ & $16.7 \pm 2.0$ & $20.3 \pm 4.9$ & 61 \\
\hline $\begin{array}{l}\text { Thalamus/ } \\
\text { Hypothalamus }\end{array}$ & $0.206 \pm 0.027$ & $0.110 \pm 0.035^{* *}$ & $25.4 \pm 1.1$ & $26.0 \pm 2.3$ & 54 \\
\hline $\begin{array}{l}\text { Colliculus/ } \\
\text { Midbrain }\end{array}$ & $0.126 \pm 0.020$ & $0.124 \pm 0.040$ & $28.0 \pm 2.5$ & $28.0 \pm 0.8$ & 99 \\
\hline Cerebellum & $0.281 \pm 0.027$ & $0.280 \pm 0.019$ & $15.8 \pm 1.1$ & $16.5 \pm 1.5$ & 104 \\
\hline
\end{tabular}

Each value represents the mean \pm SD of 3 determinations. One litter $(4 \sim 6$ rats) was used for one determination. Total GAD is the percentage of total GAD activity of microencephalic rats to that of control rats. Asterisks denote significant difference between control and microencephalic values: ${ }^{*}$ significant at $P<0.05$;** significant at $P<0.01 ; * * *$ significant at $P<0.001$.

neurotransmitters examined than a control rat, although there was no difference in the contents of them on day 21 except for a low level of aspartic acid in a microencephalic rat.

In the cerebellum, no effect of the MAMtreatment was observed except that GABA content of a 21 days old MAM-induced microencephalic rat $(0.70 \pm 0.24 \mu \mathrm{mol} / \mathrm{g}$ wet weight) was significantly lower than that of a control rat $(1.21 \pm 0.09 \mu \mathrm{mol} / \mathrm{g}$ wet weight; $P<0.01$ ).
Changes in $G A D$ activity in the brain

Cerebral GAD activity per $g$ wet weight of the tissue increased rapidly for 21 days after birth, and then increased gradually (Table II). There was no difference in GAD activity per $g$ wet weight between microencephalic and control animals. Of course the total activity per the cerebrum of a MAM-induced microencephalic rat decreased by about $50 \%$ compared with that of a control rat at all ages because cerebral weight of a microencephalic rat was 
about $50 \%$ of that of a control animal. ${ }^{1)}$

The brains of 90 days old microencephalic and control rats were divided into 6 regions, and wet weight and GAD activity were determined (Table III). Weights of cerebral regions of microencephalic rats were also about half of those of control rats in all regions, but weights of other regions, midbrain and cerebellum, were the same between two groups.

There was no difference in GAD activity per $g$ wet weight between MAM-induced microencephalic and control rats. Activity of the enzyme was highest in the colliculus and midbrain, and lowest in the cerebellum. Total activity in the cerebral cortex, nuc. caudatus/putamen, hippocampus, and thalamus/hypothalamus decreased by about $50 \%$ because of the decrease in wet weight of these regions.

\section{DISCUSSION}

Cerebral weight of a MAM-induced microencephalic rat was about half of that of a control rat " and the degree of retardation was nearly the same in the all cerebral regions (Table III). Proliferation of the cerebral nervous cells of the foetal rats was specifically inhibited by MAM administered through placenta on the 15th day of pregnancy because it was the day of division of nervous cells which should become the cerebrum. ${ }^{51}$

Protein content in the cerebrum of a microencephalic rat was $116 \pm 2 \mathrm{mg}$ and that of a control rat was $118 \pm 3 \mathrm{mg}$. There was no difference between them. On the other hand, it was reported that quantities of acetylcholinesterase, ${ }^{1)}$ monoamines (noradrenalin, dopamine and serotonine) and monoamine oxidase $^{11)}$ increased in the reduced cerebrum of a microencephalic rat. It suggests that the level of the substances direcly participating in nervous transmission increases in the reduced cerebrum for a microencephalic rat to live without hindrance. This suggestion has been confirmed by this study. A microencephalic rat showed about $40 \%$ higher contents of
GABA and other possible amino-acid neurotransmitters such as glutamic acid, glycine, aspartic acid and taurine than a control rat in the cerebrum on the day 90 after birth, although contents of these amino acids were not affected by MAM-treatment on the day 21 and in the cerebellum which is not the target organ of MAM. The result was supported by the fact that a MAM-induced microencephalic rat demonstrated a marked increase in the density of noradrenagic axons and a $9 \%$ increase in GABA content in the lateral neocortex of the cerebrum. ${ }^{12)}$ GABA and other amino acids determined in this study are listed as candidatures for possible neurotransmitters, but they have not been evident as chemical neurotransmitters except $\mathrm{GABA}^{13,14)}$ which exists specifically in the brain at a high concentration, ${ }^{15}$ is synthesized by GAD from glutamic acid, and is broken out by GABA transaminase. ${ }^{16)}$ Increase of these amino-acid neurotransmitters in the cerebrum of a 90 days old microencephalic rat may be explained as biochemical adaptability or plasticity of synapses for reduction in the number of nervous cells. On day 21 , levels of amino-acid neurotransmitters were the same between two groups; biochemical adaptation had not taken place in the cerebrum of a 21 days old microencephalic rat. An infant microencephalic rat behaved somewhat restlessly, perhaps due to the decrease in total amount of GABA that is an inhibitory neurotransmitter.

GAD activity per $g$ wet weight of the cerebrum of a microencephalic rat was not significantly different from that of a control rat (Table II). The localization of the enzyme being taken into consideration, its activity per $g$ wet weight was determined in the six regions of the brain. But there was no difference in GAD activity between microencephalic and control rats in any regions (Table III). GAD activity is generally thought to be parallel with the concentration of GABA and to be a dominant factor that controls the concentration of GABA in the brain, for distribution of GAD activity is consistent with that of GABA in the brain and GABA transaminase 
uniformally distributes in all regions of the organ. ${ }^{17 \sim 19)}$ Twenty fold augmentation of the GABA content accompanied 2 fold increase in GAD activity. ${ }^{20)}$ Assuming that the GABA content could increase in this ratio in our study, $40 \%$ increase in GABA content accounts for $2 \%$ increase in GAD activity. However, such a small increase in GAD activity was outside the limits of measurement because the standard deviations of data were more than $5 \%$ in our study.

From the experimental results, we have concluded that GABA and other possible amino-acid neurotransmitters in the cerebrum of a MAM-induced microencephalic rat augment in compensation for reduction of the cerebrum against the prediction by Moore et al. that amino-acid neurotransmitters may not be compensated in the central nervous system. $^{21 /}$ Increase of such chemical neurotransmitters as acetylcholine, monoamines and amino acid's in neurons, i.e. biochemical plasticity of neurons, has been estimated to be one of the reasons why a microencephalic rat can behave normally in spite of reduction of cerebral mass.

\section{REFERENCES}

1) Y. Nagata, Y. Nakamura and T. Watanabe, Agric. Biol. Chem., 42, 717 (1978).

2) A. Kobayashi and H. Matsumoto, Arch. Biochem., 110, 373 (1965).

3) G. L. Laqueur and H. Matsumoto, J. Natl. Cancer Inst., 37, 217 (1966).
4) G. L. Laqueur, E. G. McDaniel and H. Matsumoto, J. Natl. Cancer Inst., 39, 355 (1967).

5) M. Spatz and G. L. Laqueur, Proc. Soc. Exptl. Biol. Med., 129, 705 (1968).

6) R. K. Haddad, A. Rabe, G. L. Laqueur, M. Spatz and M. P. Valsamis, Science, 163, 88 (1969).

7) M. Sorimachi and K. Kataoka "Structure and Function of Synapses," ed. by K. Kuriyama, Ishiyaku-Shuppan Co., Tokyo, 1977, p. 69.

8) H. Matsumoto, M. Spatz and G. L. Laqueur, $J$. Neurochem., 19, 297 (1972).

9) Y. Nagata, G. Ito, M. Hibino and K. Hayashi, Nippon Nôgeikagaku Kaishi, 46, N156 (1972).

10) P. MacDonnell and O. Greengard, J. Neurochem., 24, 615 (1975).

11) Y. Nagata, J. Tanda, R. Imai and Y. Nakamura, Abstracts of papers, National Meeting of Agricultural Chemical Society of Japan, Nagoya, April, 1978, p. 444.

12) M. V. Johnston, R. Grzanna and J. T. Coyle, Science, 203. 369 (1979).

13) K. Kuriyama, B. Sisken, B. Haber and E. Roberts, Brain Res., 9, 165 (1968).

14) K. Kuriyama, B. Haber, B. Sisken and E. Roberts, Proc. Natl. Acad. Sci, 55, 846 (1966).

15) J. Awapara, A. J. Landua, R. Fuerst and B. Seale, J. Biol. Chem., 187, 35 (1950).

16) E. Roberts and H. M. Bregoff, J. Biol. Chem., 201, 393 (1953).

17) S. Fahn and L. J. Cote, J. Neurochem., 15, 209 (1968).

18) K. Kataoka, I. J. Bak, R. Hassler, J. S. Kim and A. Wagner, Exp. Brain Res., 19, 217 (1974).

19) Y. Okada, Y. Hosoya and F. Kurosawa, Jap. Acad., 53, B236 (1977).

20) C. F. Boxter "Handbook of Neurochemistry," Vol. IV, ed. by A. Lajitha, Plenum Press, N.Y., 1970, p. 289.

21) R. Y. Moore, A. Bjorklund and U. Stenevi, Brain Res., 33, 13 (1971). 